\title{
Research on Sensorless Control Strategy of PMSM based on an Improved Sliding Mode Observer
}

\author{
Suying Zhang ${ }^{1, a}$, Peidi Zhao ${ }^{2, b}$, Xue $\mathrm{Du}^{2, \mathrm{c}}, \mathrm{Ji} \mathrm{Jin}^{3}$, Huixian Liu ${ }^{4}$ \\ ${ }^{1}$ School of Hebei, Science and Technology University, Shijiazhuang 050000, China \\ ${ }^{2}$ School of Hebei, Science and Technology University, Shijiazhuang 050000, China \\ bhbstu02_zpd@163.com
}

\begin{abstract}
A sensor-less control method of permanent magnet synchronous motor (PMSM) based on sliding mode observer (SMO) is studied in the synchronous rotating reference orthogonal coordinate system in the work. The SMO is proposed under the synchronous rotating coordinate system and in which the sign function is replaced by saturation function in order to eliminate the chattering phenomenon. The rotor position and speed estimation can be obtained from the induced electromotive force (EMF) observed from SMO by using the phase locked loop (PLL), which can achieve the purpose of sensor-less control. The simulation results show that the system can accurately track the rotor position information in a real time and meet the needs of the actual motor control performance.
\end{abstract}

Keywords: PMSM, sensor-less control, SMO, saturation function, PLL.

\section{Introduction}

PMSM is widely used in industrial production due to its superior features such as simple structure, lower loss and high efficiency. In order to achieve a smooth start and high-performance control effect, the three-phase PMSM must obtain accurate rotor position and speed information [1, 2]. Normally, the rotor speed and position can be measured by encoder or a mechanical sensor, which not only increase the cost, size and weight of the system, but also limit the application of PMSM in some special occasions [3]. The sensor-less control technology overcomes these defects and has been paid more and more attention in the last few years.

SMO is robust to parameter variations, external disturbances and has low requirement on model precision compared with other algorithms of sensor-less control. This article proposes a control method under the synchronization reference frame. The sign function in SMO is replaced by saturation function to eliminate the chattering phenomenon caused by SMO. Finally the computer simulation results demonstrate the proposed method can meet the needs of the actual electric machine control performance commendably.

\section{Mathematical model of PMSM}

The vector control mathematical model of PMSM is established under the synchronization reference frame. And the stator current equation is given by

$$
\left\{\begin{array}{l}
\frac{d i_{d}}{d t}=\frac{1}{L_{d}}\left(-R i_{d}+u_{d}+L_{q} i_{q} \omega_{e}-E_{d}\right) \\
\frac{d i_{q}}{d t}=\frac{1}{L_{q}}\left(-R i_{q}+u_{q}+L_{d} i_{d} \omega_{e}-E_{q}\right)
\end{array}\right.
$$

where $i_{d}$ and $i_{q}$ are the stator current of $\mathrm{d}$ axis and $\mathrm{q}$ axis respectively; $u_{d}$ and $u_{q}$ are the stator voltage of $\mathrm{d}$ and q axisrespectively; $L_{d}$ and $L_{q}$ are the inductance of $\mathrm{d}$ and q axis respectively; $\omega_{e}$ is the rotorvelocity. Under the synchronousrotating coordinate frame, it is assumed that $E_{d}=0, E_{q}=\omega_{e} \psi_{f}$, where $\psi_{f}$ is the rotor permanent magnet flux. 


\section{Design of sliding mode observer}

In order to obtain the value of induced EMF in Eq.(1), the SMO can be designed as

$$
\begin{aligned}
& \left\{\begin{array}{l}
\frac{d \hat{i_{d}}}{d t}=\frac{1}{L_{d}}\left(-R \hat{i_{d}}+u_{d}+L_{q} \hat{i_{q}} \omega_{e}-V_{d}\right) \\
\frac{d \hat{i_{q}}}{d t}=\frac{1}{L_{q}}\left(-R \hat{i_{q}}+u_{q}+L_{d} \hat{i_{d}} \omega_{e}-V_{q}\right)
\end{array}\right. \\
& \left\{\begin{array}{l}
V_{d}=k \operatorname{sgn}\left(\hat{i_{d}}-i_{d}\right) \\
V_{q}=k \operatorname{sgn}\left(\hat{i_{q}}-i_{q}\right)
\end{array}\right.
\end{aligned}
$$

Where $\hat{i_{d}}$ and $\hat{i_{q}}$ are the stator current observation of $\mathrm{d}$ and $\mathrm{q}$ axis respectively and $k$ is the sliding mode gain.

The current error state equation of system can be obtained by Eq.(2) subtracting Eq.(3), described as in vector form

$$
\frac{d \tilde{i}}{d t}=A \tilde{i}+B(-V+E)
$$

Where $\tilde{i}=\hat{i}-i$, specifically, $\tilde{i}=\left[\begin{array}{cc}\tilde{i_{d}} & \tilde{i_{q}}\end{array}\right]^{T}, V=\left[\begin{array}{ll}V_{d} & V_{q}\end{array}\right]^{T}, E=\left[\begin{array}{ll}E_{d} & E_{q}\end{array}\right]^{T}$, the coefficient is given by $A=\left[\begin{array}{cc}-\frac{R}{L_{d}} & \frac{L_{q}}{L_{d}} \omega_{e} \\ -\frac{L_{d}}{L_{q}} \omega_{e} & -\frac{R}{L_{q}}\end{array}\right], B=\left[\begin{array}{cc}\frac{1}{L_{d}} & 0 \\ 0 & \frac{1}{L_{q}}\end{array}\right]$.

The sliding surface function of SMO is defined as $\tilde{i}=\left[\begin{array}{cc}\tilde{i}_{d} & \tilde{i_{q}}\end{array}\right]^{T}=0$. The system enters the sliding mode when $k$ is large enough and at the same time $\tilde{i}=d \tilde{i} / d t=0$ is tenable.

The induced EMF can be rewritten as

$$
E=\left[k \operatorname{sgn}\left(\hat{i_{d}}-i_{d}\right) \quad k \operatorname{sgn}\left(\hat{i_{q}}-i_{q}\right)\right]^{T}
$$

Eq.(5) shows that the induced EMF contains discontinuous high frequency signal. The equivalent quantity can be expressed as follows after the signal is processed through a low-pass filter

$$
E=\left[k \operatorname{sgn}\left(\hat{i_{d}}-i_{d}\right) \quad k \operatorname{sgn}\left(\hat{i_{q}}-i_{q}\right)\right]_{e q}^{T}=\left[\begin{array}{ll}
0 & \omega_{e} \psi_{f}
\end{array}\right]^{T}
$$

The expression of sliding mode gain can be described as $k=n \max \left[\frac{E_{d}}{L_{d}} \operatorname{sgn}\left(\tilde{i_{d}}\right)-\left(\frac{R}{L_{d}}+\frac{L_{d}}{L_{q}} \omega_{e}\right)\left|\tilde{i_{d}}, \frac{E_{q}}{L_{q}} \operatorname{sgn}\left(\tilde{i_{q}}\right)-\left(\frac{R}{L_{q}}+\frac{L_{q}}{L_{d}} \omega_{e}\right)\right| \tilde{i_{q}} \mid\right]$ when the SMO enters the sliding mode, where $n$ is an invariant constant.

The block diagram of the traditional SMO is shown in Fig.1. 


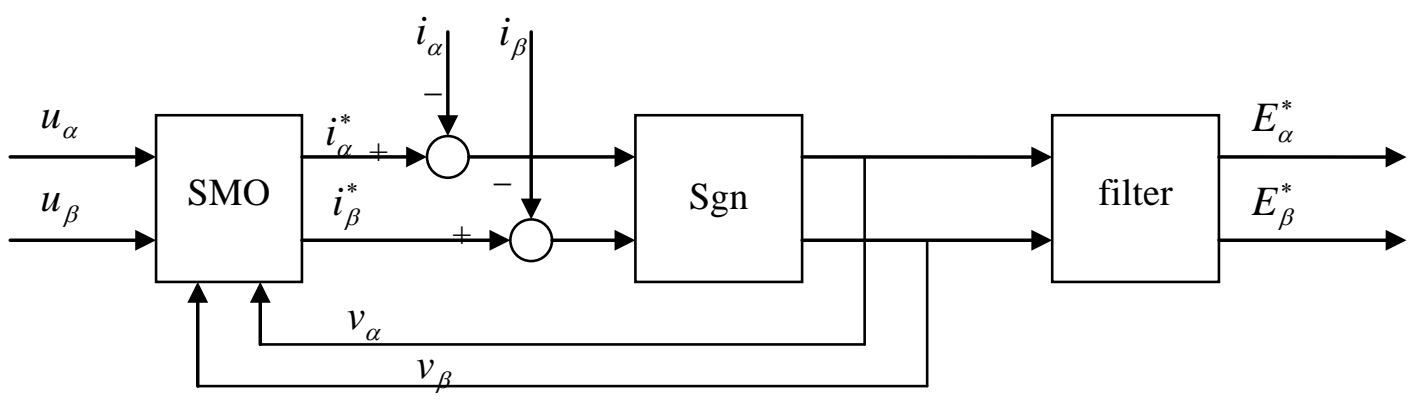

Fig.1 Block diagram of traditional SMO

As mentioned above, there will be high frequency chattering phenomenon because of the discontinuity of the control, which has a great influence on the stability of the system [4]. In order to improve the performance, the switching function in the traditional is replaced by saturation function [5] which is described as $\operatorname{sat}(s)=\left\{\begin{array}{l}1, s>\varepsilon \\ k s,|s| \leq \varepsilon, \frac{1}{\varepsilon} \\ -1, s<-\varepsilon\end{array}\right.$, where $\varepsilon$ represents the boundary layer.

\section{Rotor position estimation based on PLL}

As stated earlier, the purpose of the design is to obtain the estimation of induced electromotive force. Eq. (1) shows that the rotor speed can be obtained from the calculations on q axis induced EMF. The $\mathrm{q}$ axis estimation of rotor speed and rotor position angle estimation are given by $\hat{\omega}_{e}=\frac{v_{q}}{\psi_{f}}, \hat{\theta}_{e}=\int \hat{\omega}_{e} d t$

The accuracy of estimation has strong dependence on the motor parameters. In this work, the position and velocity of rotor are estimated from the induced EMF by using PLL structure to improve the accuracy and the stability of the system.

The three-phase symmetrical sinusoidal phase voltage of PMSM is defined as

$$
\left\{\begin{array}{l}
u_{a}=u \cos \omega_{e} t \\
u_{b}=u \cos \left(\omega_{e} t-2 \pi / 3\right) \\
u_{c}=u \cos \left(\omega_{e} t+2 \pi / 3\right)
\end{array}\right.
$$

where $\omega_{e}=p_{n} \pi n / 30, \theta_{e}=\omega_{e} t$, and $u, p_{n}, n, \omega_{e}, \theta_{e}$ are the phase voltage amplitude, pole-pair number of the motor, mechanical speed, the rotor speed, and the instantaneous rotor speed.

The $d-q$ axis voltage equation can be obtained through the transformation matrix, described as

$$
\left[\begin{array}{l}
V_{d} \\
V_{q}
\end{array}\right]=\frac{1}{3}\left[\begin{array}{lll}
\cos \left(\theta_{e}\right) & \cos \left(\theta_{e}-2 \pi / 3\right) & \cos \left(\theta_{e}+2 \pi / 3\right) \\
-\sin \left(\theta_{e}\right) & -\sin \left(\theta_{e}-2 \pi / 3\right) & -\sin \left(\theta_{e}+2 \pi / 3\right)
\end{array}\right]\left[\begin{array}{l}
u_{a} \\
u_{b} \\
u_{c}
\end{array}\right]
$$

In order to improve the simulation accuracy, the corresponding discrete state equations of SMO current equation by using reverse difference method is described as

$$
\left\{\begin{array}{l}
\hat{i_{d}}(k+1)=A \hat{i_{d}}(k)+B\left[u_{d}(k)-V_{d}(k)+\omega_{e} L_{q} i_{q} \hat{(k)}\right] \\
\hat{i_{q}}(k+1)=A^{\prime} \hat{i_{q}}(k)+B^{\prime}\left[u_{q}(k)-V_{q}(k)+\omega_{e} L_{d} i_{d} \hat{(k)}\right]
\end{array}\right.
$$

where the Fourier Series Expression of $\exp \left(A T_{s}\right)$ is given by as follows and $T_{s}$ is the sample time

$$
\exp \left(A T_{s}\right)=1+A T_{s}+\frac{\left(A T_{s}\right)^{2}}{2}+\frac{\left(A T_{s}\right)^{3}}{3 !}+\cdots
$$


The coefficient in Eq. (14) can be rewritten as

$$
A=\exp \left(-R / L_{d} T_{s}\right), B=\frac{1}{R}(1-A), A^{\prime}=\exp \left(-R / L_{q} T_{s}\right), B^{\prime}=\frac{1}{R}\left(1-A^{\prime}\right)
$$

\section{Simulation modeling and analysis}

It is assumed that the rotor of the PMSM is permanent magnet, and the flux linkage is constant. The specifications of the PMSM are $R=0.011 \Omega ; \mathrm{L}_{\mathrm{d}}=1.6 \mathrm{mH}, L_{q}=1 \mathrm{mH} ; \psi_{f}=0.077 \mathrm{~Wb}$; the rated voltage and motor intertie are set as $311 \mathrm{~V} 、 0.8 \mathrm{e}-3 \mathrm{Kg} \cdot \mathrm{m}^{2}$ respectively, and the number of poles is 3 .

The simulation model of the system is created in MATLAB/Simulink software. The parameters of the current loop controller , the speed loop controller and the parameters of PLL are set to respectively $k_{p i_{d}}=32, k_{i i_{d}}=220 ; k_{p i_{q}}=20, k_{i i_{q}}=220 ; k_{p N}=0.02, k_{i N}=1 ; k_{p}=30, k_{i}=450$. The speed reference is set as $N_{\text {ref }}=700 \mathrm{r} / \mathrm{min}$.

Fig.2 shows the $\mathrm{d}$ axis and $\mathrm{q}$ axis induced electromotive force curve obtained by SMO.

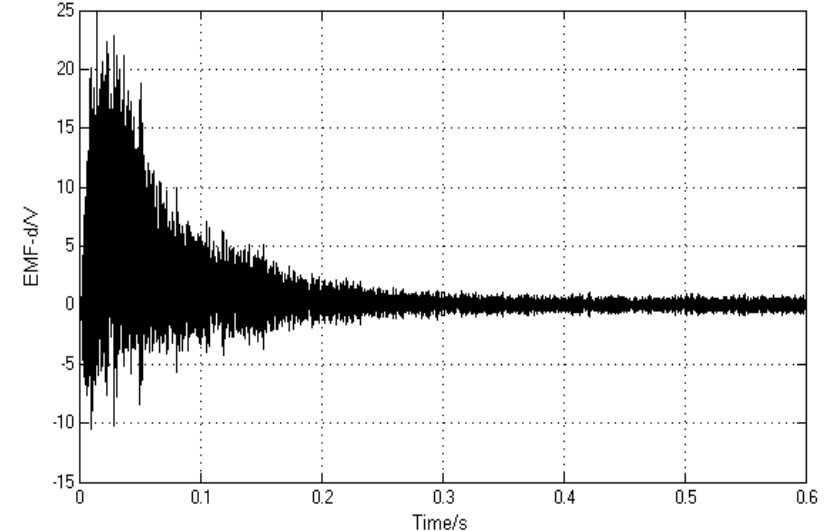

a) Induced electromotive force of $d$ axis

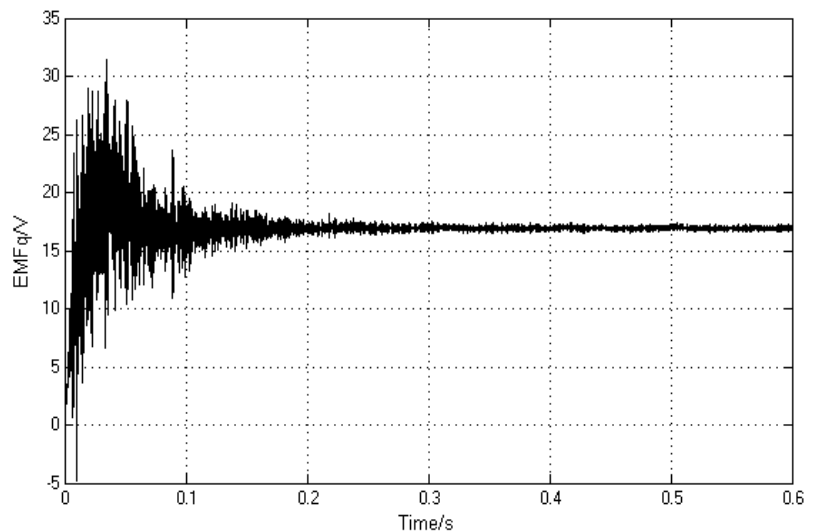

b) Induced electromotive force of q axis

Fig.2 Induced electromotive force

The rotor speed and position angle are estimated by PLL in the modified SMO. And Figs.3 and 4 shows the error of rotor speed and position angle by PLL and conventional calculation method.

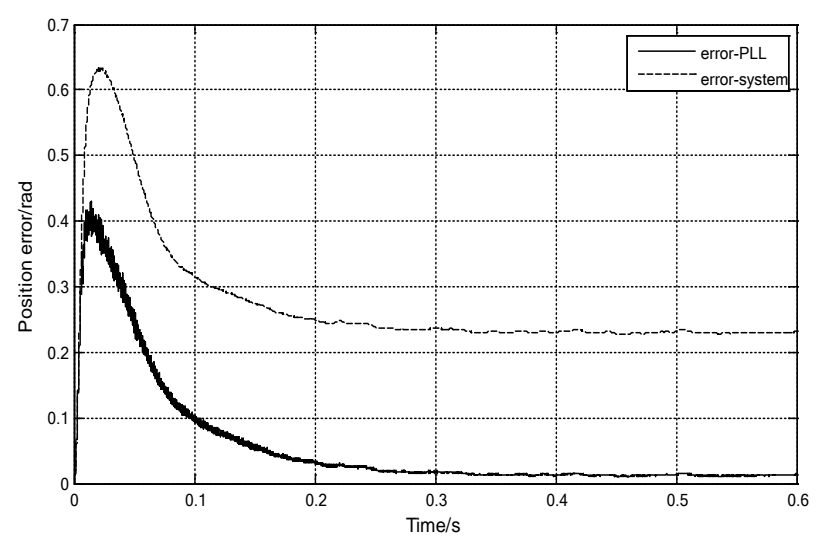

Fig.3 Rotor position error 


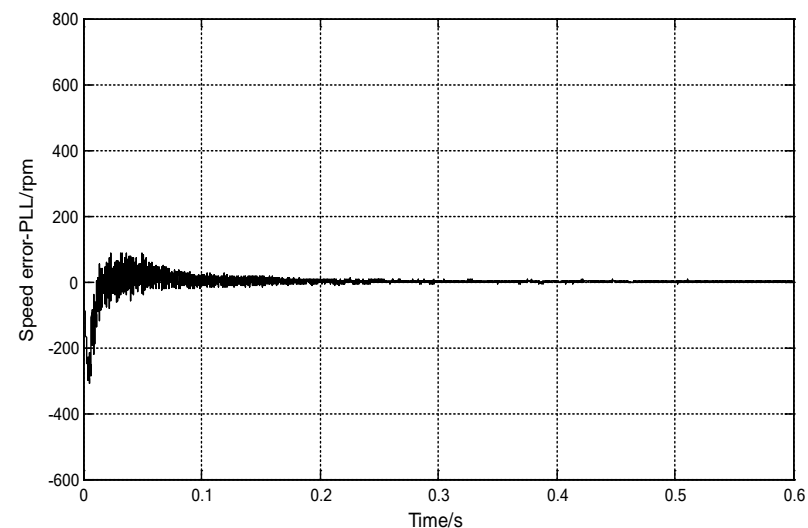

a) Rotor speed error-PLL

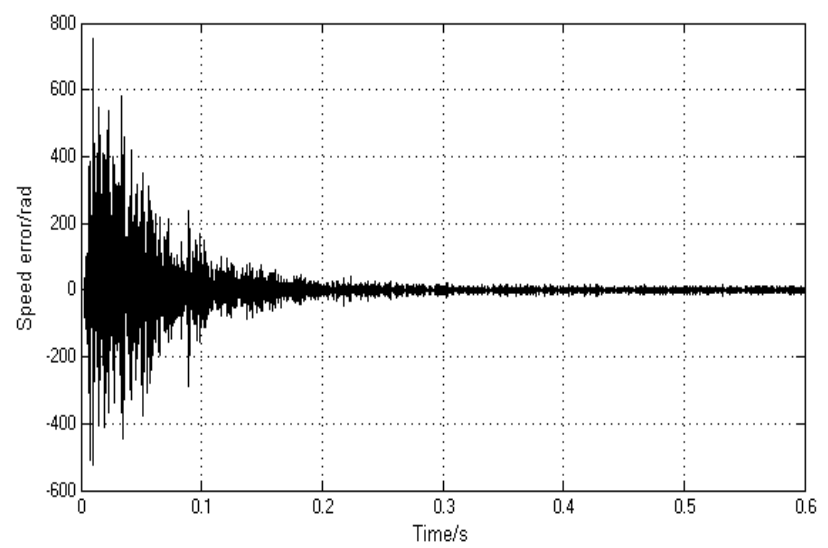

b ) Rotor speed error

Fig.4 Rotor position and speed error

The simulation results show that the error of the speed and position angle is larger during the rotate speed rising, while the error is smaller when the motor tends to be stable and the motor speed and position angle based on PLL has smaller error and higher precision compared with the conventional SMO. Then on this foundation, the next task in the work is to presents a modified PLL to reduce the chattering phenomenon and make the dynamic performance better.

\section{Acknowledgments}

This work was financially supported by Natural Science Foundation of China (51507048).

\section{References}

[1] Wang Hanxiao, Liu Ling, Wu Weihua. A Sensor-less Permanent Magnet Synchronous Motor Control Strategy for Improved Sliding Mode Observers with Stator Parameters Identification [J]. Journal of Xi'an Jiaotong University, 2016, 50(6):104-109.

[2] Chen Siyi, Pi Youguo. Position Sensorless Control for Permanent Magnet Synchronous Motor Based Mode Observer and Sliding Mode Controller [J]. Transactions of China Electrotechnical Society, 2016, 31 (12):108-117.

[3] Preindl M, Schaltz E. Sensor-less model predictive direct current control using novel second-order PLL observer for PMSM drive system [J]. IEEE Transactions on Industrial Industrial Electronics, 2011, 58 (9):4087-4095.

[4] Sun Fangfang, Huang Xianghui, Chen Ke. Sensor-less Control Strategy for Permanent Magnet Synchronous Motor of Improved Sliding Mode Observer[J], Explosion-proof Electric Machine,2017,52(194):1-5.

[5] Zhang Runbo, Lin Rongwen, Gao Jingkai. Research On Sensor-less Control Method of PMSM based on an Improved Sliding Mode Observer [J], Electrical Technology, 2016, 1:51-61.

[6] Yuan Lei, Hu Bingxin, Wei Keyin, et al. Modern permanent magnet synchronous motor control theory and MATLAB simulation [M], Bei Jing: Beijing University of Aeronautics and Astronautics Press, 2016:5-8. 